\title{
A Bacterial Continuous Culture System Based on a Microfluidic Droplet Open Reactor
}

\author{
Manami Ito, ${ }^{* 1}$ Haruka Sugiura, ${ }^{* 1}$ Shotaro Ayukawa, ${ }^{* 2}$ Daisuke KIGA, ${ }^{* 1, * 3}$ and \\ Masahiro TAKInOUE $* 1, * 4 \dagger$ \\ *1 Department of Computational Intelligence and Systems Science, Tokyo Institute of Technology, \\ 4259 Nagatsuta-cho, Midori, Yokohama, Kanagawa 226-8502, Japan \\ *2 Education Academy of Computational Life Sciences (ACLS), Tokyo Institute of Technology, \\ 4259 Nagatsuta-cho, Midori, Yokohama, Kanagawa 226-8502, Japan \\ *3 Earth-Life Science Institute (ELSI), Tokyo Institute of Technology, 2-12-1 Ookayama, Meguro, \\ Tokyo 152-8550, Japan \\ *4 PRESTO, Japan Science and Technology Agency (JST), 4-1-8 Honcho, Kawaguchi, Saitama 332-0012, \\ Japan
}

\begin{abstract}
Recently, micrometer-sized bacterial culture systems have attracted attention as useful tools for synthetic biology studies. Here, we present the development of a bacterial continuous culture system based on a microdroplet open reactor consisting of two types of water-in-oil microdroplets with diameters of several hundred micrometers. A continuous culture was realized the through supply of nutrient substrates and the removal of waste and excess bacterial cells based on repeated fusion and fission of droplets. The growth dynamics was controlled by the interval of fusion. We constructed a microfluidic system and quantitatively assessed the dynamics of the bacterial growth using a mathematical model. This system will facilitate the study of synthetic biology and metabolic engineering in the future.
\end{abstract}

Keywords Continuous culture, cell density control, nonequilibrium open system, synthetic biology, genetic engineering, droplet microfluidics, water-in-oil microdroplet, emulsion, nonlinear dynamics, metabolic engineering

(Received September 30, 2015; Accepted October 21, 2015; Published January 10, 2016)

\section{Introduction}

In recent years, micrometer-sized bacterial culture systems have become increasingly important in the field of synthetic biology. ${ }^{1}$ Such culture systems have been developed based on microfluidic technologies, ${ }^{2}$ and have contributed to the investigation of artificial gene circuits in bacterial cells in a steady-state environment, ${ }^{3,4}$ or in a dynamical environment with external stimulation. ${ }^{5,6} \quad$ Unlike conventional beaker-sized continuous culture systems, microfluidic continuous culture systems enable the real-time microscopic observation of individual bacterial cells during cell culture and subsequent control of bacterial cells based on information obtained from real-time observations. In addition, microfluidic continuous culture systems can dramatically reduce the volume of culture media (to less than several milliliters per day) compared with the volume of beakersized continuous culture systems (i.e., more than several liters per day). Although microfluidic continuous culture systems have such advantages, in previously developed microfluidic continuous culture systems, control of the entire medium solution in the microchannel system was needed to realize the observation and control of cultured bacterial cells. Therefore,

$\dagger$ To whom correspondence should be addressed.

E-mail: takinoue.m.aa@m.titech.ac.jp simpler microfluidic technologies for bacterial continuous culture systems are required.

In this study, we propose a simple microscale bacterial continuous culture system based on droplet microfluidic technologies..$^{7-12}$ Here, we used a droplet open reactor ${ }^{13,14}$ for constructing a bacterial continuous culture system (Fig. 1). The droplet open reactor consisted of two types of water-in-oil (W/O) microdroplets with diameters of several hundred micrometers (Fig. 1a). The first type was a reactor droplet fixed in a microchannel; the reactor droplet contained bacteria and the culture medium. The second type was carrier droplets containing a fresh culture medium for the bacteria (i.e., including a sufficient amount of nutrient substrates); the carrier droplets were produced at a T-junction in the microchannel and passed through the microchannel with an oil flow (Figs. 1a and 1b). The carrier and reactor droplets did not fuse in the absence of an alternative current (AC) voltage because the W/O microdroplets were stabilized using a surfactant. In contrast, they fused through electric-field-induced dynamic instability when an AC voltage was applied. ${ }^{13-16}$ When the carrier and reactor droplets fused, a fresh culture medium was partly supplied to the reactor droplet; also, the bacteria and waste in the reactor droplet were partly ejected. Note that the entire solution in the reactor droplet was not exchanged in a single fusion-fission event. The reactor and carrier droplets were monitored at the area of the reactor droplet (Fig. 1c), and the fusion of the droplets was controlled 
a
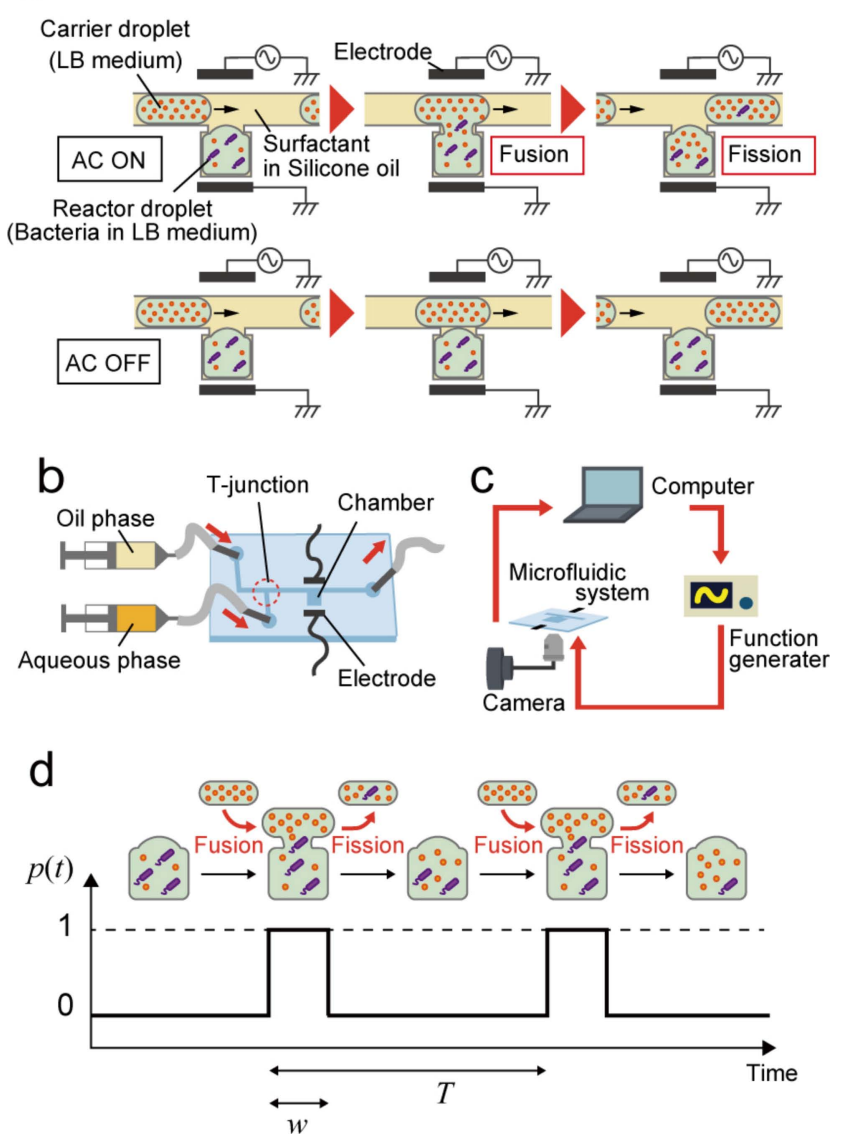

Fig. 1 Bacterial continuous culture system based on a droplet open reactor. (a) The mechanism of the supply of substrates and the ejection of excess bacterial cells based on the fusion and fission of the reactor and carrier droplets. (b) Image of the entire microfluidic system. (c) Control of the fusion based on the observation of the droplets and the computer program. (d) The AC voltage application profile for the control of the fusion.

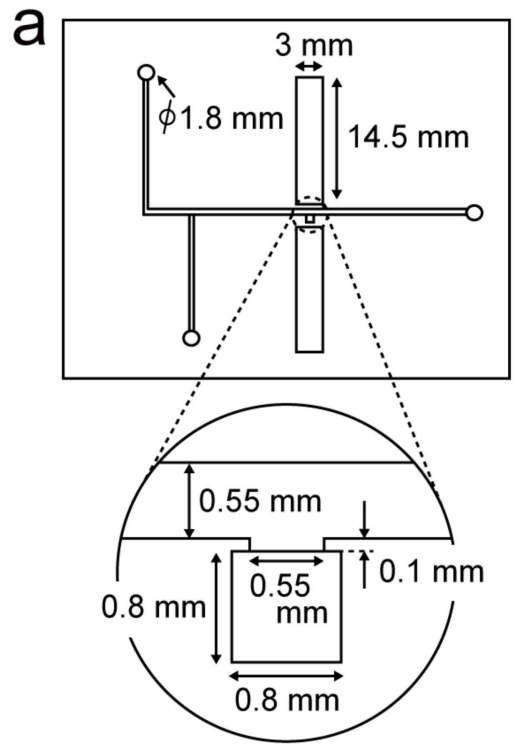

by a fusion-control program based on monitoring information (Figs. 1c and 1d). In the present work, we designed and applied this type of droplet open reactor for a micrometer-sized bacterial continuous culture system. In addition, we propose a mathematical model for a bacterial continuous culture. We believe that this system can be applied to the construction of a microchemostat, and can also promote studies in the biological sciences, including synthetic biology.

\section{Experimental}

\section{Fabrication of a microfluidic system}

The design of the microfluidic system is shown in Fig. 2a, and the constructed microfluidic system is shown in Figs. 2b and 2c. The microfluidic system was constructed of two poly(methyl methacrylate) (PMMA) plates (1 mm thick; extruded PMMA; Mitsubishi Rayon, Tokyo, Japan). The microchannel was designed using three-dimensional (3D) computer-aided design (CAD) software (Rhinoceros 3D; McNeel, Seattle, WA) and was fabricated on the upper plate using a fine milling machine (MDX-40A; Roland DG, Shizuoka, Japan) with a 0.5-mm diameter end-mill (MAEM230-0.5; NS Tool, Tokyo, Japan). The upper plate had three holes for two inlets and an outlet and two square holes for electrodes; additionally, it had one more hole at the top of the square chamber $(0.8 \times 0.8 \times 1 \mathrm{~mm})$ for the injection of a reactor droplet containing bacterial cells in the culture medium (Fig. 2a). The upper and lower plates were attached by thermal compression bonding for approximately $30 \mathrm{~min}$ at $90^{\circ} \mathrm{C}$ (AL194-L; Romanov). Three Teflon tubes (internal diameter: $0.5 \mathrm{~mm}$; TGK, Tokyo, Japan) were attached to the holes and fixed with Araldite (AR-R30; NICHIBAN, Tokyo, Japan). At the beginning of the experiments, a reactor droplet containing bacterial cells in culture medium was introduced into the square chamber through the top injection hole using a micropipette; the hole was then sealed with a transparent cellophane tape (CT-15S2P; NICHIBAN). The oil phase and aqueous phase were then transported through the system using microsyringe pumps (LEGATO180; KD Scientific,

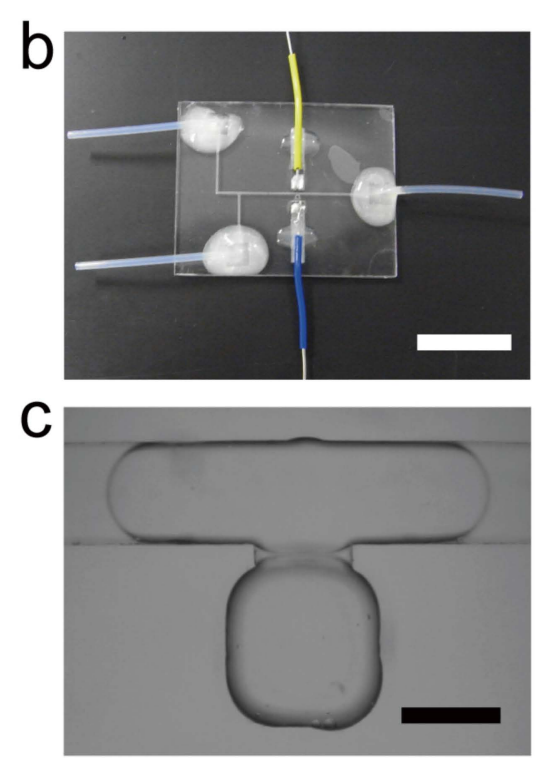

Fig. 2 Design of the microfluidic system and the constructed microfluidic system. (a) Design of the microfluidic system. (b) Image of the entire constructed microfluidic system. Scale bar, $2 \mathrm{~cm}$. (c) Enlarged view of the reactor droplet and the carrier droplet. Scale bar, $500 \mu \mathrm{m}$. 

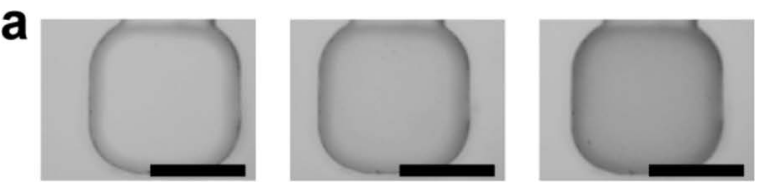

$2.0 \times 10^{7}$ cells mL-1 $8.0 \times 10^{8}$ cells $\mathrm{mL}^{-1} \quad 2.0 \times 10^{9}$ cells $\mathrm{mL}^{-1}$

b
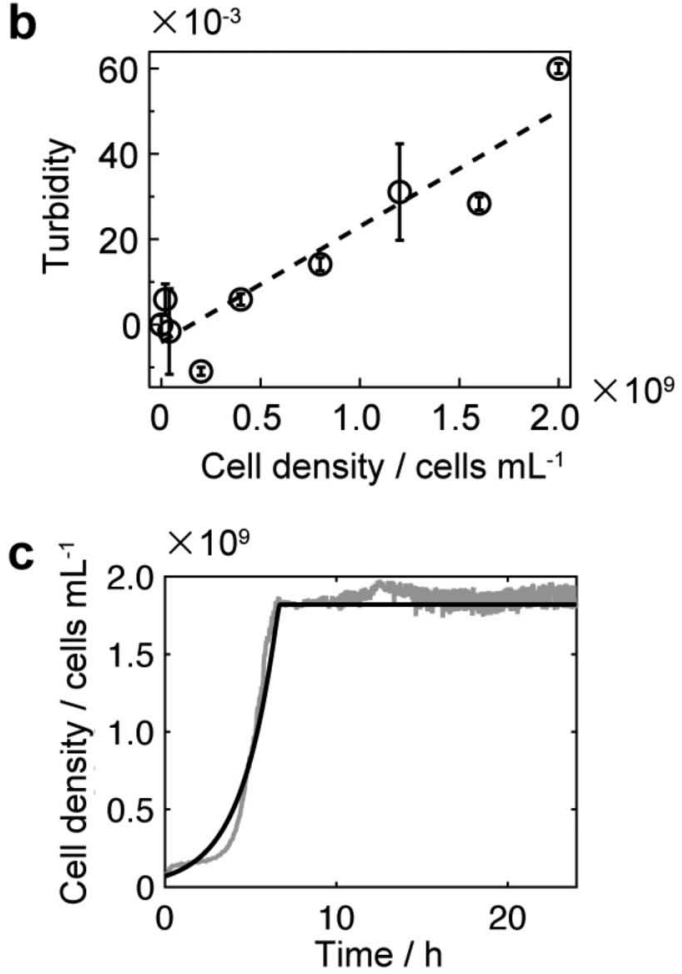

Fig. 3 Optical measurements of the bacterial cell density and a bacterial batch culture. (a) Microscopic images of the square chamber containing E. coli cell solutions. Scale bar, $500 \mu \mathrm{m}$. (b) Relationship between the turbidity $\log _{10}\left(I_{0} / I\right)$ and cell density of E. coli. (c) Time course of a batch culture of $E$. coli in the reactor droplet. The cell density was calculated from the turbidity using the relationship shown in (b).

Holliston, MA) and disposable syringes $(20 \mathrm{~mL}$; SS-20ESZ; Terumo, Tokyo, Japan) connected to the microfluidic device by Teflon tubes (internal diameter: $0.5 \mathrm{~mm}$; TGK). The electrodes were connected to a function generator (WF1973; NF Corp., Yokohama, Japan) through a voltage amplifier (M-2629B-2CH; MESS-TEK, Wako, Saitama, Japan). An AC voltage was applied, and a single fusion and fission event was realized during the time when the $\mathrm{AC}$ voltage was switched on; this was controlled using an image-processing module of the OpenCV library and Microsoft Visual C++ (Microsoft Corp., Redmond, WA).

Optical measurements of bacterial cell density under a brightfield microscope

Bacterial cell densities were measured based on the turbidity of the bacterial solutions (Fig. 3a); increased turbidity indicated an increase in the density of bacterial cells. First, Escherichia coli (JM2.300 strain with pSB6A1-Ptet-GFP, a BioBrick plasmid that constitutively expresses a green fluorescent protein $)^{17}$ was cultured overnight at $37^{\circ} \mathrm{C}$ in an $\mathrm{LB}$ medium (Nacalai Tesque, Kyoto, Japan) containing antibiotics (50 $\mu \mathrm{g} \mathrm{mL}^{-1}$ ampicillin; Nacalai Tesque, Kyoto, Japan). The optical density at $600 \mathrm{~nm}\left(\mathrm{OD}_{600}\right)$ of the 5-fold-diluted overnightcultured solution was measured using an absorption spectrophotometer (V-630BIO, JASCO, Tokyo, Japan), and then $\mathrm{OD}_{600} \sim 0.6-0.8$. The overnight-cultured solution was diluted 100 -fold with the LB medium containing antibiotics, and the number of $E$. coli cells was counted using a cell counter plate (2-7124-01; ASONE, Osaka, Japan) to determine the cell density of E. coli. Finally, serial dilutions of overnight-cultured solution were introduced into the chamber of the microfluidic system. The intensities of the transmitted light of the bacterial solution in the chamber $(I)$ and the transmitted light at the circumference of the chamber $\left(I_{0}\right)$ were measured using a brightfield microscope (IX71; Olympus, Tokyo, Japan) and a digital camera (EOS 60D; Canon, Tokyo, Japan), and were then analyzed using an ImageJ. Finally, the turbidity was calculated as $\log _{10}\left(I_{0} / I\right)$. Numerical analyses were performed using software, MATLAB (MathWorks, Natick, MA).

\section{Bacterial batch culture and continuous culture in the reactor droplet}

The oil phase was a silicone oil (KF-96L-1CS; Shin-Etsu Chemical, Tokyo, Japan), with a $2 \%$ (w/w) surfactant (KF-6028; Shin-Etsu Chemical). The aqueous phase of the reactor droplet introduced into the square chamber was the E. coli culture solution (the 100-fold diluted solution of an overnight culture of E. coli). The aqueous phase of the carrier droplets was the LB medium containing antibiotics. The flow rates of the oil and the aqueous phases were both set at $8.8 \mu \mathrm{L} \mathrm{min}{ }^{-1}$. For a batch culture in the reactor droplet, no $\mathrm{AC}$ voltage was applied; i.e., the carrier and reactor droplets did not fuse. For a continuous culture in the reactor droplet, $300 \mathrm{~V}$ (peak-to-peak; $1 \mathrm{kHz}$ ) of an AC voltage was applied with an interval of $T$; under these conditions, the fusion duration, $w$, was approximately $1.5 \mathrm{~s}$. The temperature was set at $37^{\circ} \mathrm{C}$ using a thermoplate (MATS-U52RA26; Tokai Hit, Shizuoka, Japan). Images were taken every 2 min for $72 \mathrm{~h}$ using an optical microscope (IX71) and a digital camera (EOS 60D), and then analyzed using an ImageJ. The turbidity was analyzed as described above for the optical measurements. Numerical analyses were performed using MATLAB.

\section{Numerical simulations}

Numerical simulations were performed using MATLAB.

\section{Results and Discussion}

\section{Construction of a microfluidic system}

We constructed a microfluidic system for a bacterial continuous culture based on a droplet open reactor (Fig. 2). Figure $2 \mathrm{a}$ shows the detailed design of the microfluidic system. Figure $2 b$ is an image of the fabricated microfluidic system. The reactor droplet was fixed in the square chamber in the microchannel (Fig. 2c).

Microscopic optical measurements of bacterial batch culture in the reactor droplet without the medium feeding

First, we established a method to measure the density of E. coli cells in the solution based on the optical turbidity. Figure 3a shows bright-field microscopy images of the square chambers containing E. coli. The turbidity increased as the density of the $E$. coli cells increased. We defined the turbidity as $\log _{10}\left(I_{0} / I\right)$ (see details in the Experimental section). The turbidity was proportional to the cell density when it was greater than approximately $0.4 \times 10^{9}$ cells $\mathrm{mL}^{-1}$ (Fig. 3 b). 
Next, we analyzed the growth curves of the E. coli batch culture in the reactor droplet (i.e., the reactor droplet did not fuse with the carrier droplets containing the fresh culture medium; gray solid line in Fig. 3c). In this batch culture, the cell density saturated at approximately $8 \mathrm{~h}$. The growth curve analysis showed that the reactor droplet could be used for a bacterial culture.

To obtain the kinetic parameters of $E$. coli cell growth in the reactor droplet, the following mathematical model for the batch culture was constructed, ${ }^{18}$ and fitted to the log phase of the growth in the experimental data (Fig. 3c; $0-6$ h):

$$
\begin{aligned}
& \frac{\mathrm{d} c(t)}{\mathrm{d} t}=g(t) c(t), \\
& \frac{\mathrm{d} s(t)}{\mathrm{d} t}=-\frac{1}{Y} \frac{\mathrm{d} c(t)}{\mathrm{d} t}, \\
& g(t)=\frac{g_{\max } s(t)}{K+s(t)},
\end{aligned}
$$

where $c(t), s(t)$, and $g(t)$ are the cell density, the apparent substrate concentration, and the cell growth rate in the reactor droplet, respectively; $Y$ is the yield of cells per apparent substrate concentration; $g_{\max }$ and $K$ indicate the maximum value of the cell growth rate and the Michaelis constant of the MichaelisMenten-type model, respectively. The Michaelis-Menten-type model for the cell growth rate (Eq. (3)) indicates that the growth rate is proportional to the apparent substrate concentration when the substrate concentration is low $(<K)$; in contrast, the growth rate is saturated to the maximum value $\left(g_{\max }\right)$ when the substrate concentration is high $(>>K)$. In Fig. 3c, the black solid line was a fitted curve produced by fitting Eqs. (1) - (3) to the gray solid line. From this analysis, we obtained the values of the numerical parameters, $Y \times s_{0}=1.75 \times 10^{9}$ cells $\mathrm{mL}^{-1}, \mathrm{~K} / \mathrm{s}_{0}=$ 0.01 , and $g_{\max }=0.5 \mathrm{~h}^{-1}$, where $s_{0}$ is the initial apparent substrate concentration in the reactor droplet; i.e., $s_{0}=s(0)$. In the numerical model, $Y$ and $K$ cannot be obtained independently from $s_{0}$. These parameters were used in the numerical simulations shown below.

Experimental investigation of bacterial continuous culture in the droplet open reactor

Figure 4 shows the results of an E. coli continuous culture in the droplet open reactor that allows nutrient supply during the culture. In all cases, the cell densities increased to a greater extent than the saturation cell density in the batch culture (Fig. 3c), suggesting that the damage to the bacterial cells from the application of AC voltage was negligible. The cell growth rate was slightly higher when the fusion interval was $60 \mathrm{~s}$ than when the fusion interval was $30 \mathrm{~s}$. This suggested that, in the case of the frequent fusion ( $T=30 \mathrm{~s})$, the supply of substrates in the medium was sufficient, but the flow rate of an $E$. coli dilution droplet was fast, compared to the case of $T=60 \mathrm{~s}$.

Figure $4 \mathrm{e}$ shows sequential images of the droplet fusion and fission when $E$. coli was cultured with a fusion interval $T$ of $30 \mathrm{~s}$. The bacterial growth had a spatial heterogeneity in the reactor droplet, likely because the mixing of the nutrient substrate after droplet fusion was not instantaneous, and bacterial movement in the reactor and ejection from the reactor was slower than the substrate molecules.

Numerical analyses of the bacterial continuous culture in the droplet open reactor

To analyze the bacterial continuous culture in the droplet open

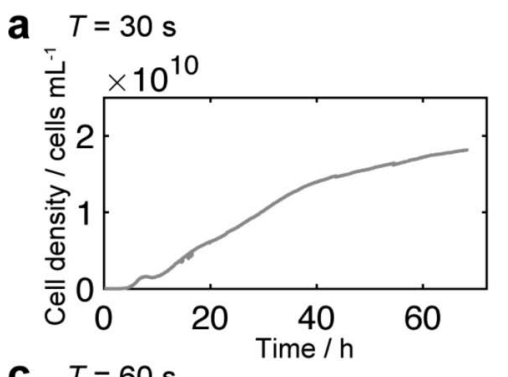

b $T=30 \mathrm{~s}$
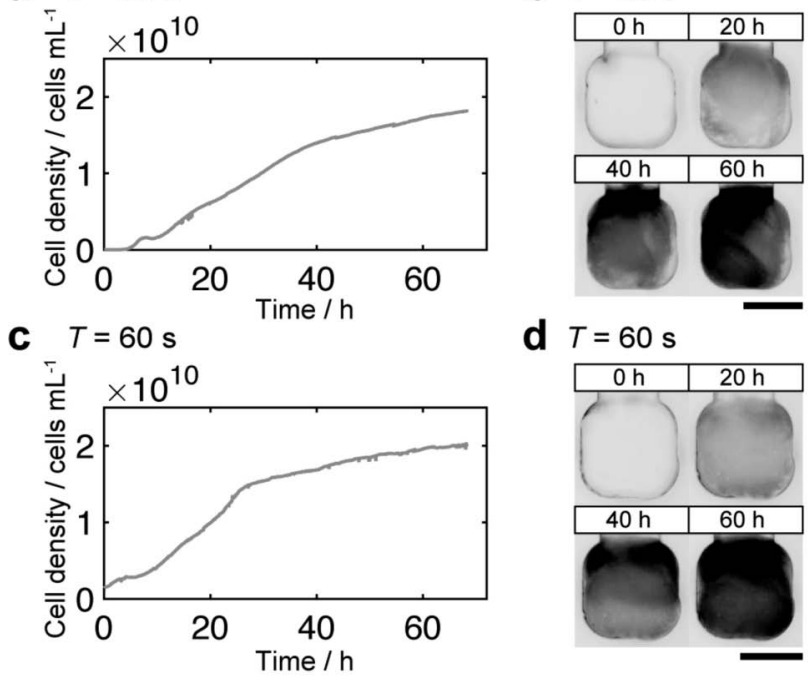

d $T=60 \mathrm{~s}$

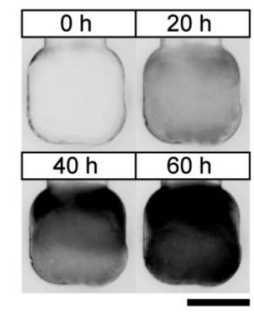

e

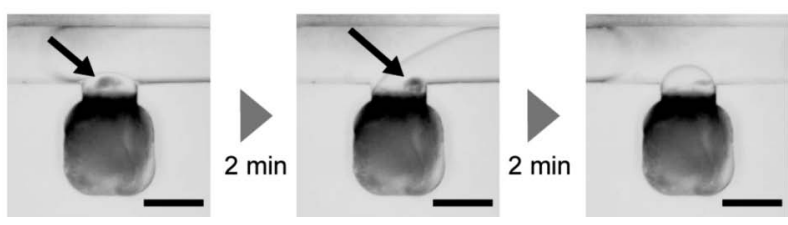

Fig. 4 Bacterial continuous culture in the reactor droplet. (a) and (c) Time courses of $E$. coli continuous culture for fusion intervals of $T=$ 30 and $60 \mathrm{~s}$, respectively. In these experiments, we estimated the cell density based on the standard curve shown in Fig. $3 b$ by assuming the linearity of the standard curve, although the cell density was higher than the range of the standard curve. (b) and (d) Microscopic images at the time $0,20,40$, and $60 \mathrm{~h}$ in the continuous culture experiments (a) and (c), respectively. Scale bars, $500 \mu \mathrm{m}$. (e) Sequential images of the droplet fusion and fission when E. coli was cultured under the conditions of $T=30 \mathrm{~s}$. Black arrows indicate an aggregation of $E$. coli. Scale bars, $500 \mu \mathrm{m}$. A movie showing the results in (e) is provided as supporting information.

reactor, we constructed an extended mathematical model of a bacterial continuous culture in the droplet open-reactor system based on Eqs. (1) - (3), a general nonlinear dynamics model of bacterial continuous culture in centimeter/meter-sized reactors (Fig. 5a). ${ }^{18}$

$$
\begin{aligned}
& \frac{\mathrm{d} c_{1}(t)}{\mathrm{d} t}=g_{1}(t) c_{1}(t)-D_{\mathrm{c} 1} p(t) c_{1}(t), \\
& \frac{\mathrm{d} s_{1}(t)}{\mathrm{d} t}=-\frac{g_{1}(t)}{Y} c_{1}(t)+D_{\mathrm{s} 1} p(t)\left(s_{0}-s_{1}(t)\right), \\
& g_{1}(t)=\frac{g_{\max } s_{1}(t)}{K+s_{1}(t)}, \\
& \frac{\mathrm{d} c_{2}(t)}{\mathrm{d} t}=g_{2}(t) c_{2}(t)+D_{\mathrm{c} 2}\left(c_{1}(t)-c_{2}(t)\right), \\
& \frac{\mathrm{d} s_{2}(t)}{\mathrm{d} t}=-\frac{g_{2}(t)}{Y} c_{2}(t)+D_{\mathrm{s} 2}\left(s_{1}(t)-s_{2}(t)\right), \\
& g_{2}(t)=\frac{g_{\max } s_{2}(t)}{K+s_{2}(t)},
\end{aligned}
$$

where the definitions of $c(t), s(t), g(t), Y, g_{\max }$, and $K$ are the same 
a
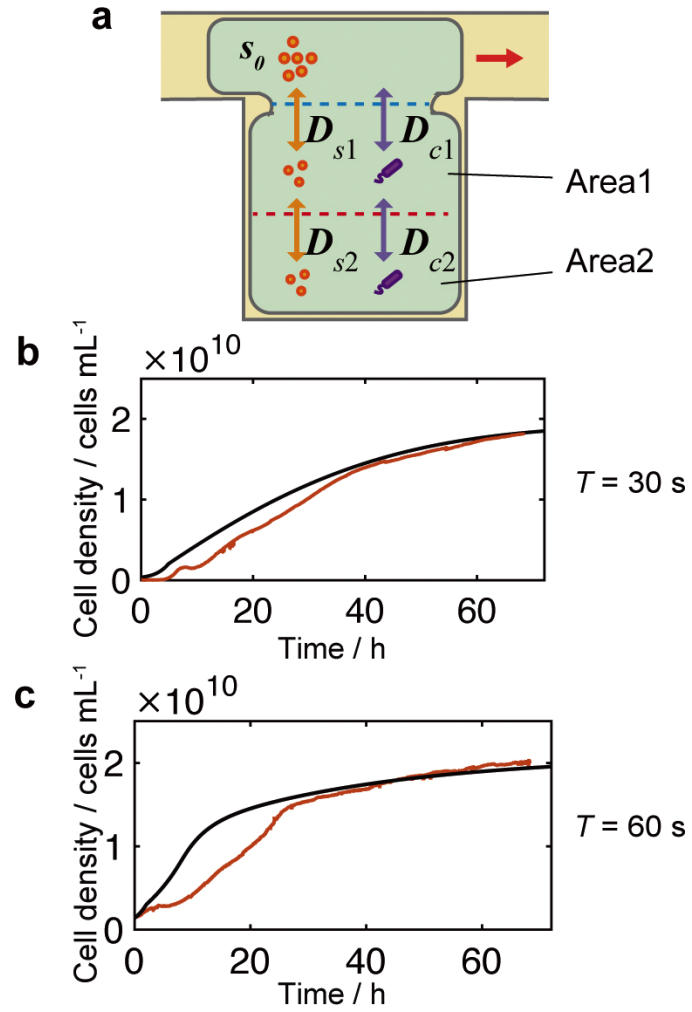

Fig. 5 Numerical analyses of bacterial continuous culture based on the droplet open reactor. (a) Conceptual illustration of the mathematical model (Eqs. (4)-(9)). (b) and (c) Comparison of numerical simulations and the experimental results for fusion intervals of $T=$ 30 and $60 \mathrm{~s}$, respectively. $w=1.5 \mathrm{~s}$. Black solid line: numerical simulation data (average cell density, $\left.\left(c_{1}(t)+c_{2}(t)\right) / 2\right)$. Red solid line: experimental results.

as those in Eqs. (1) - (3); $s_{0}$ is the initial apparent substrate concentration in the reactor droplet $\left(s_{0}=s(0)\right)$ and the apparent substrate concentration in the carrier droplets; and $D_{\mathrm{c}}$ and $D_{\mathrm{s}}$ are the exchange rates of cells and substrates during the fusion, respectively. In this model, we assume that the reactor is divided into two areas, as shown in Fig. 5a. This assumption is based on an observation that the cell growth behaviors differed between the areas near and far from the droplet fusion position (Fig. 4e). Indexes 1 and 2 of $c(t), s(t), g(t), D_{\mathrm{c}}$, and $D_{\mathrm{s}}$ indicate the areas 1 and 2 shown in Fig. 5a. $p(t)$ expresses a fusionfission time course (Fig. 1d); $p(t)=1$ when the reactor and carrier droplets fuse, and $p(t)=0$ when they do not fuse. By mathematical analysis, we found that $p(t)$ is approximately $w / T,{ }^{14}$ where $T$ and $w$ are the fusion interval and the fusion duration, respectively. Therefore, we have

$$
D_{\mathrm{c} 1} p(t) \sim D_{\mathrm{c} 1} \frac{w}{T} \equiv \bar{D}_{\mathrm{c} 1} \quad \text { and } \quad D_{\mathrm{s} 1} p(t) \sim D_{\mathrm{s} 1} \frac{w}{T} \equiv \bar{D}_{\mathrm{s} 1}
$$

where $\bar{D}_{\mathrm{c} 1}$ and $\bar{D}_{\mathrm{s} 1}$ are the average exchange rates of the cells and substrates, respectively.

The black lines in Figs. $5 b$ and $5 c$ show the numerical simulation results. In the simulations, we used the experimentally measured parameters $\left(Y \times s_{0}, K / s_{0}\right.$, and $\left.g_{\max }\right)$, and assumed that $D_{\mathrm{s} 1}=2, \quad D_{\mathrm{s} 2}=D_{\mathrm{s} 1} / 200, \quad D_{\mathrm{c} 1}=D_{\mathrm{s} 1} / 12, \quad D_{\mathrm{c} 2}=D_{\mathrm{c} 1} / 300 . \quad$ These results showed that the mathematical model approximately reproduced the tendency of the experimental results, although the reproducibility was not perfect. The disagreement may have resulted from the dynamically formed heterogeneity of the cell density in the reactor droplet (Figs. $4 \mathrm{~b}$ and $4 \mathrm{~d}$ ) and the discrete ejection of aggregated cells from the reactor droplet (Fig. 4e), because these factors were not perfectly represented in the numerical model.

\section{Conclusions}

In this study, we developed a microfluidic bacterial continuous culture system based on a droplet open reactor. The nutrient conditions of a continuous culture were controlled by the fusion and fission of microdroplets. Due to the continuous culture, the cell density greatly increased compared to the batch culture. In addition, the fusion interval changed the growth curve of bacteria. In terms of the control method of supply and ejection of substrates and cells, this system is simpler than previously developed microfluidic continuous culture systems in terms of the control method of the supply and ejection of substrates and cells because the supply and ejection rates were controlled by the $\mathrm{AC}$ voltage alone, without requiring control of the entire solution in the microchannel, the use of syringes, or other complicated processes. Moreover, the growth curve was explained by a mathematical model, although the predictability was not perfect; by tuning the numerical parameters and improving the model further, we will be able to improve the predictability of the growth dynamics. In this study, large microdroplets with diameters of several hundred micrometers caused the spatial heterogeneity of bacterial cell growth in the reactor, enhancing the complexity of the growth dynamics. By decreasing the sizes of microdroplets to approximately several tens of micrometers, the issue will be resolved. Furthermore, this microfluidic system can be applied to a micrometer-sized chemostat that can control the bacterial cell density in a reactor. The control of the bacterial cell density, for example, will be helpful to study cell diversification, depending on the cell density. ${ }^{19}$ Therefore, our method will contribute to a wide range of fields, such as synthetic biology and metabolic engineering.

\section{Acknowledgements}

We thank Prof. Hiroyuki Noji, Prof. Kazuhito Tabata, and Prof. Shoji Takeuchi (Univ. Tokyo) for helpful discussions. This research was supported by PRESTO (Design and Control of Cellular Functions) from JST to M. T., a Grant-in-Aid for Challenging Exploratory Research (No. 26540150) from JSPS to M. T., a Grant-in-Aid for Scientific Research on Innovative Areas (No. 23119005) from MEXT to D. K., and a Research Strategy Office Grant (B) Interdisciplinary Research Support for Young Scientists from Tokyo Tech to D. K. and M. T.

\section{Supporting Information}

A movie showing the results in Fig. 4e is provided as Supporting Information. The movie was produced at 7200 -fold the speed of real time. This material is available free of charge on the Web at http://www.jsac.or.jp/analsci/.

\section{References}

1. M. R. Bennett and J. Hasty, Nat. Rev. Genet., 2009, 10, 628. 
2. R. Rusconi, M. Garren, and R. Stocker, Ann. Rev. Biophys., 2014, 43, 65.

3. F. K. Balagadde, L. You, C. L. Hansen, F. H. Arnold, and S. R. Quake, Science, 2005, 309, 137.

4. F. K. Balagaddé, H. Song, J. Ozaki, C. H. Collins, M. Barnet, F. H. Arnold, S. R. Quake, and L. You, Mol. Syst. Biol., 2008, 4, 187

5. M. R. Bennett, W. L. Pang, N. A. Ostroff, B. L. Baumgartner, S. Nayak, L. S. Tsimring, and J. Hasty, Nature, 2008, 454, 1119.

6. J. Stricker, S. Cookson, M. R. Bennett, W. H. Mather, L. S. Tsimring, and J. Hasty, Nature, 2008, 456, 516.

7. M. Takinoue and S. Takeuchi, Anal. Bioanal. Chem., 2011 400, 1705.

8. S.-Y. Teh, R. Lin, L.-H. Hung, and A. P. Lee, Lab Chip, 2008, 8, 198.

9. M. T. Guo, A. Rotem, J. A. Heyman, and D. A. Weitz, Lab Chip, 2012, 12, 2146.

10. A. D. Griffiths and D. S. Tawfik, Trends Biotechnol., 2006, 24,395 .
11. A. Huebner, S. Sharma, M. Srisa-Art, F. Hollfelder, J. B. Edel, and A. J. Demello, Lab Chip, 2008, 8, 1244.

12. H. Song, D. L. Chen, and R. F. Ismagilov, Angew. Chem., Int. Ed., 2006, 45, 7336.

13. M. Takinoue, H. Onoe, and S. Takeuchi, Small, 2010, 6, 2374.

14. H. Sugiura, M. Ito, T. Okuaki, Y. Mori, H. Kitahata, and M. Takinoue, Nat. Commun., 2015, 6, 10212.

15. C. Priest, S. Herminghaus, and R. Seemann, Appl. Phys. Lett., 2006, 89, 134101.

16. S. Herminghaus, Phys. Rev. Lett., 1999, 83, 2359.

17. Registry of Standard Biological Parts (iGEM BioBrick), http://parts.igem.org/Main_Page.

18. H. L. Smith and P. Waltman, "The Theory of the Chemostat: Dynamics of Microbial Competition", 2008, Cambridge University Press, Cambridge, England.

19. R. Sekine, M. Yamamura, S. Ayukawa, K. Ishimatsu, S. Akama, M. M. Takinoue, M. Hagiya, and D. Kiga, Proc. Natl. Acad. Sci. U. S. A., 2011, 108, 17969. 